\title{
A Biomechanical Comparison of 3.5 Locking Compression Plate Fixation to 3.5 Limited Contact Dynamic Compression Plate Fixation in a Canine Cadaveric Distal Humeral Metaphyseal Gap Model
}

\author{
By \\ Dean Eric Filipowicz \\ Thesis submitted to the Faculty of the Virginia Polytechnic Institute and State University \\ in partial fulfillment of the requirements for the degree of \\ Master of Science in Biomedical and Veterinary Sciences \\ Approval Committee: \\ Chair: Otto I Lanz, DVM, Diplomate ACVS \\ Ronald M McLaughlin, DVM, Diplomate ACVS \\ Colin B Carrig, BVSc, PhD, Diplomate ACVR
}

May 30, 2008

Blacksburg, Virginia

Keywords: Fracture Model, Axial Compression, Cyclic Torsion, LC-DCP, LCP

Copyright 2008, Dean E. Filipowicz 


\title{
A Biomechanical Comparison of 3.5 Locking Compression Plate Fixation to 3.5 Limited Contact Dynamic Compression Plate Fixation in a Canine Cadaveric Distal Humeral Metaphyseal Gap Model
}

\author{
By
}

Dean Eric Filipowicz

\begin{abstract}
Objective- To compare the biomechanical properties of 3.5 locking compression plate (LCP) fixation to 3.5 limited contact dynamic compression plate (LC-DCP) fixation in a canine cadaveric, distal humeral metaphyseal gap model in static axial compression and cyclic axial compression and torsion.

Study Design- Biomechanical in vitro study.

Sample Population-30 paired humeri from adult, medium to large breed dogs.

Methods- Testing was performed monotonically to failure in axial compression on ten pairs of humeri, cyclically in axial compression for 10,000 cycles on ten pairs and cyclically in torsion for 500 cycles on the last ten pairs.

Results- Humeral constructs stabilized with LCPs were significantly stiffer than those plated with LC-DCPs when loaded in axial compression ( $\mathrm{P}=0.0004)$. When cyclically loaded in axial compression over 10,000 cycles, the LC-DCP constructs were significantly stiffer than those constructs stabilized with LCPs $(\mathrm{P}=0.0029)$. Constructs plated with LC-DCPs were significantly more resistant to torsion over 500 cycles than those plated with LCPs $(\mathrm{P}<0.0001)$, though no difference was detected during the first 280 cycles.
\end{abstract}


Conclusions- The increased stiffness of LCP constructs in monotonic loading compared to constructs stabilized with non-locking plates may be attributed to the stability afforded by the plate-screw interface of locking plates. The LCP constructs demonstrated less stiffness in dynamic testing in this model, likely due to plate-bone offset secondary to non-anatomic contouring and occasional incomplete seating of the locking screws when using the torque-limiting screw driver.

Clinical Relevance- LCPs yield less stiff fixation under dynamic loading than conventional LC-DCPs when applied to severely comminuted, metaphyseal fractures. Improving anatomical contouring of the plate and insuring complete screw insertion into the locking plate hole may improve stiffness when using LCPs in comminuted fractures. 


\section{ACKNOWLEDGEMENTS}

Grant Information: The author would like to recognize the Virginia Veterinary Medical Association Pet Memorial Fund as well as Synthes Vet for financial support.

Acknowledgements: The author would like to recognize Ms. Pam Arnold for her acquisition of specimens and laboratory expertise and Dr. Steve Elder for his invaluable assistance in manufacturing and implementing the testing jig, in helping design the testing protocol, and in helping perform the testing. The author would additionally like to thank Dr. Steven Werre for his assistance with statistical analyses.

The author would additionally like to acknowledge the members of his committee and surgical department who contributed immensely during his present training. 


\section{TABLE OF CONTENTS}

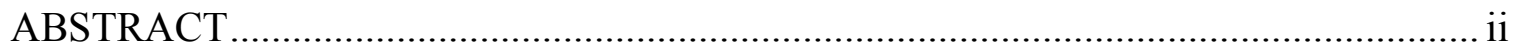

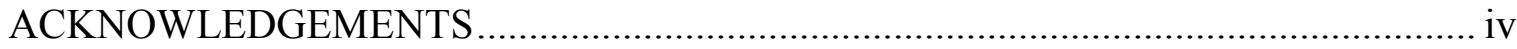

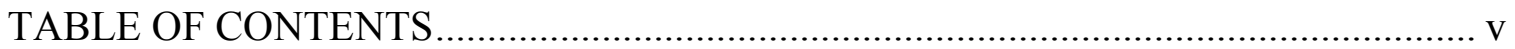

CHAPTER I: Project Rationale and Literature Review ……………………………...... 1

A: Problem Definition and Current Management.................................................... 1

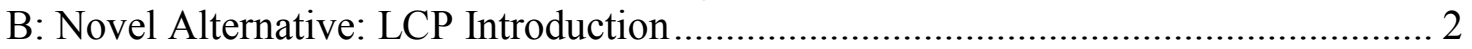

C: Literature Review of Biomechanical Testing .................................................... 3

D: Initial Clinical Results................................................................................. 7

CHAPTER II: A Biomechanical Comparison of 3.5 Locking Compression Plate Fixation to 3.5 Limited Contact Dynamic Compression Plate Fixation in a Canine Cadaveric Distal Humeral Metaphyseal Gap Model ................................................................. 10

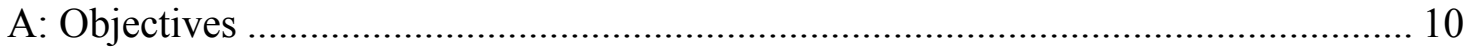

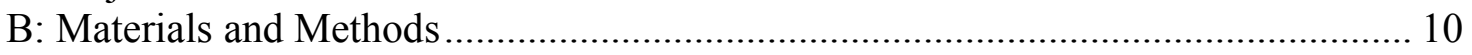

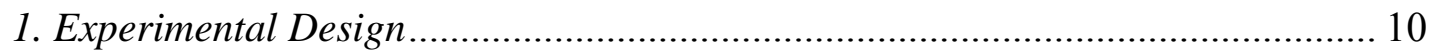

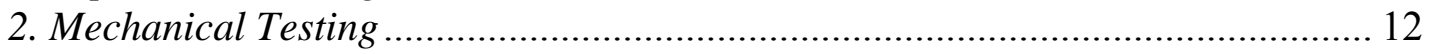

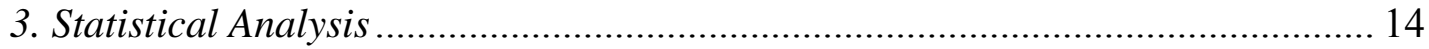

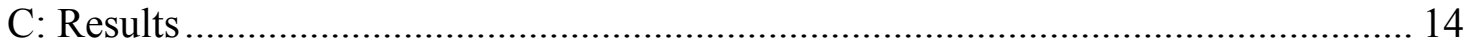

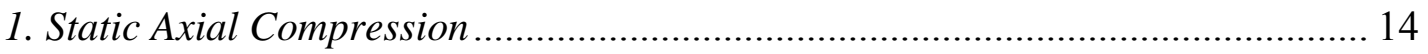

2. Cyclic Axial Compression........................................................................ 15

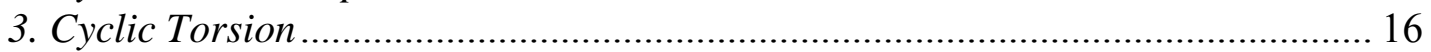

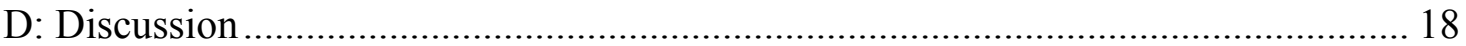

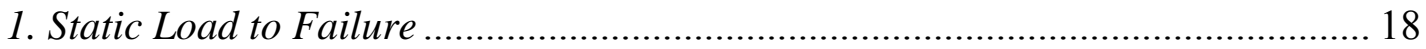

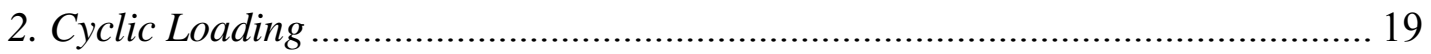

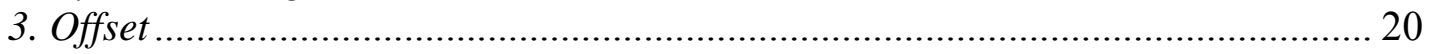

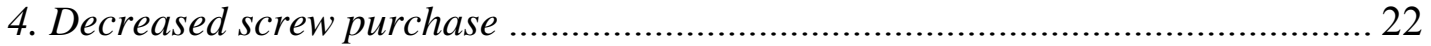

5. Possible incomplete seating ………………………........................................ 23

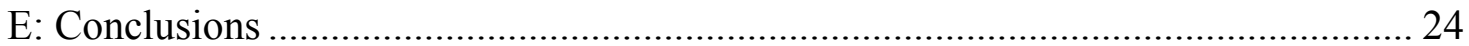

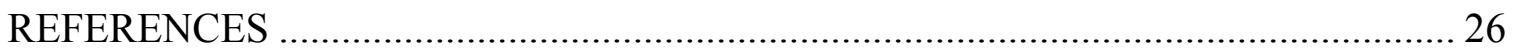

APPENDIX 1: FIGURES ...................................................................................

Figure 1: Locking compression plate (LCP). Note the locking screw on the left and

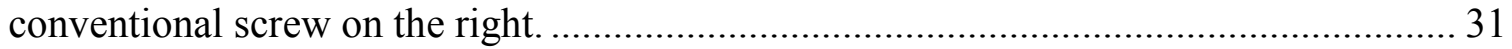

Figure 2: Dynamic Compression Unit (DCU) of the LCP. The non-threaded section on the left accepts conventional, non-locking screws, and the threaded section on the right accepts locking screws.

Figure 3: Application of the LCP. Note the threaded drill guide screwed into the proximal aspect of the plate and the $1.5 \mathrm{Nm}$ torque limiter being used during screw

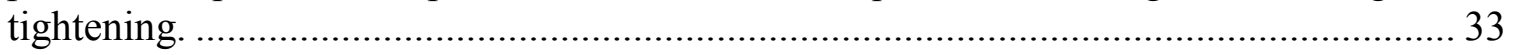

Figure 4: Representative LC-DCP construct during axial testing. ................................... 34

Figure 5: Load vs. Deformation curve of representative humeral pair loaded monotonically in axial compression. ................................................................................ 35

Figure 6: Mean resistance to torsional loading over 500 cycles...................................... 36 
Figure 7: Distal portion of LCP construct after testing. Note the offset present between the plate and bone between the two screws. Note also how the proximal screw does not penetrate the trans cortex.

Figure 8: Illustration depicting an exaggeration of the loss of the buttressing of the bone on the underside of the plate and the exposed portions of the screws secondary to a contiguous offset. 38 Figure 9: LCP construct showing incomplete seating of the proximal of the distal screw heads. 


\section{CHAPTER I: Project Rationale and Literature Review}

\section{A: Problem Definition and Current Management}

The outcome of highly comminuted, metaphyseal fracture repairs in small animal surgery is too often uncertain. Unlike diaphyseal fractures where fixation can straddle the comminution, metaphyseal fractures provide limited and usually compromised bone substrate for implant purchase. Current fixation options for this latter type of fracture have limitations. External fixators are often successfully employed in similar human fractures, but the extensive post-operative care necessary and the fragility of the exposed hardware make this method of fixation perhaps less consistently suitable for small animal patients. Interlocking nails are very useful if the anti-rotational screws or studs can align with solid bone, but this is a rare occurrence in metaphyseal fractures, rarer still if the fracture happens to be comminuted. Arguably, the best chance of success in veterinary cases with comminuted, metaphyseal fractures currently lies with plates or plate/rod combinations. Unfortunately, conventional plating has inherent deficiencies when applied to this type of fracture. ${ }^{1}$

Currently used orthopaedic plates (dynamic compression plates (DCP), limited contact dynamic compression plates (LC-DCP), etc.) must be solidly compressed to bone. This compression, accomplished by screw tightening, must be such that the frictional force generated between plate and bone is sufficient throughout healing to withstand all loads not supported by the bone itself. To help achieve this compression, plates are contoured to match the bone surface. The increased contact, however, comes at the

expense of the periosteal blood supply. ${ }^{2}$ Inadequate quantity or quality of bone stock such 
as found in metaphyseal or comminuted fractures, or in the immature or geriatric patient, often prevent appropriate compression as well as maintenance of reduction, both during the repair and later, during loading. As a result, conventional plating repairs in these situations are often tenuous. A new plating system with inherent stability stemming from the screw head/plate interface however may obviate some of the shortcomings in these types of fractures and ideally ensure a more substantial fixation.

\section{B: Novel Alternative: LCP Introduction}

Human orthopaedists have been enjoying the benefits of locking compression plates (LCPs) since their introduction eight years ago ${ }^{3}$, and veterinary surgeons are beginning to take notice. This plating system differs from conventional ones in that threads on the head of locking screws match those tapped during manufacturing in the plate holes; locking occurs once the screw heads are tightened into the plate. Please see Figure 1. The plate/screw interface creates a rigid construct with angular and axial stability. The plate is laid on the bone instead of being compressed to it. The periosteal blood supply is then preserved, and contouring no longer must be anatomic. This latter benefit equates to a less aggressive surgical approach as well as decreased concern over loss of reduction during fixation or loading. The additional stability may also allow for engagement of fewer cortices per fragment, making locking plates particularly suited for metaphyseal and comminuted fractures and in situations where only one cortex is available. ${ }^{4-6}$

Though locking technology may prove beneficial for the management of such fractures, indications for non-locking plates still exist. Articular fractures and 
osteotomies, for example, require an adherence to the tenets of plating osteosynthesis, namely rigid fixation and anatomic reduction, as do instances in which the blood supply is already compromised and bone is already necrotic. ${ }^{2,7}$ What's more, conventional compression plates such as the dynamic compression plate and the limited contact dynamic compression plate can provide interfragmentary compression and lag screw application. Plates with pure locking holes can not.

A plating system that could incorporate the advantages of biological osteosynthesis (callus formation through minimal but appropriate amounts of relative motion, vascular and soft tissue preservation) but could also be used in plating osteosynthesis applications would allow a surgeon an intra-operative choice of techniques without having to maintain two plating systems. Such a system would also be useful for those fractures that called for a synergy of both management schools. The LCP is such a system. A conically threaded plate hole was merged with the dynamic compression unit of an LC-DCP to form a combi-hole. Locking screws can be placed through the more than 200 degree threaded section of this hole to provide angular and axial stability. Nonlocking screws can be placed through the DCU portion of the hole to create interfragmentary compression or be used in lag fashion, and can be angled similarly to screws in conventional plates. ${ }^{7,8}$ Please see Figure 2.

\section{C: Literature Review of Biomechanical Testing}

Before veterinary orthopaedists can adopt this new technology and replace current plating systems, biomechanical testing must be performed at least to compare LCPs to today's standard. There are currently only two papers which contrast the biomechanical properties of the LCP to other fracture stabilization methods in veterinary orthopaedics; 
the first uses an equine model $^{9}$ with double plating and the second a small animal fracture model $^{10}$. In the former, Florin et al. concluded that in an equine, oblique fracture model, constructs double plated with orthogonally placed LCPs were stiffer, had a higher yield strength, and provided the least movement at the fracture when tested in four point bending compared to constructs stabilized with DCPs or LC-DCPs in the same configuration or clamp rod internal fixators (CRIFs). It was this group's conclusion that the stabilization provided by all three plates tested was likely sufficient to withstand postoperative loading, but the LCP may still be preferable for its potentially superior biomechanical characteristics and its greater reliance on biological osteosynthesis. Aguila et al. performed an in vitro biomechanical comparison of a canine diaphyseal gap model buttressed with either LCPs or LC-DCPs. ${ }^{10}$ Static four point bending and torsional tests revealed no difference in stiffness between constructs in this femoral diaphyseal model stabilized with three screws per fragment on contoured plates. The LC-DCP constructs showed significantly more torsional resistance at three of five points when tested cyclically to failure, but failure occurred at lower twist angles than their locked counterparts. It was concluded that the not dissimilar biomechanical characteristics of the LCPs and LC-DCPs allowed the former to substitute for the latter in diaphyseal fractures, and that the biological benefits afforded by the former may justify their additional cost.

A search of the human literature yields similar conclusions from a small number of reports. Gardner et al. compared LCPs to LC-DCPs in a radial diaphyseal gap model and did not find a significant difference in initial normalized stiffness or change in normalized stiffness over the course of cyclic four point bending and cyclic torsion tests 
between constructs stabilized with either plate. ${ }^{11}$ Overall survival was also found to be similar between groups subjected to bending, but constructs stabilized with LCPs survived significantly longer than the LC-DCP specimens in torsional testing. A study by Korner et al comparing distal humeral metaphyseal gap models double plated with either LCPs or LC-DCPs found no significant difference in stiffness in static four point bending and torsion, in dynamic four point bending, and in strength to failure in four point bending provided the double plating configuration was the same. ${ }^{12}$

Fulkerson et al. used an LCP in an osteoporotic, synthetic, ulnar, diaphyseal, gap model to evaluate biomechanical differences between constructs stabilized with locking screws or conventional non-locking screws. ${ }^{13}$ During cyclic, axial, elastic compression, constructs stabilized with bicortical locked screws showed 15 times less gap closure than unicortically locked constructs and 25 times less displacement than non-locked plates. The same bicortical locked plates then withstood more than 160 times more cycles to failure in cyclical cantilever bending than either the unicortically locked plates or the non-locking ones; that failure of these latter two groups did not occur at significantly different cycles may suggest that pre-stressed constructs stabilized with unicortical locking screws resist fatigue as well as conventional plates. Though Fulkerson et al. did all testing on LCPs, it is not unreasonable to assume an LC-DCP would have provided similar data to Fulkerson's LCPs with non-locking screws.

Biomechanical studies highlighting the increased stability of other locking plates are increasing in number and may lend additional support to the use of LCPs. A recent study comparing locking plates to an identical plate with non locking screws in a proximal humeral gap model found that constructs stabilized with the locking plate failed 
at a greater load and demonstrated increased stiffness in static axial loading tests. ${ }^{14}$ These constructs also showed greater resistance to fatigue and less maximum deformation at failure after cyclic axial loading. Stoffel et al found that intra-articular calcaneal fractures stabilized with locking plates showed less irreversible deformation after 1000 cycles and sustained a greater maximum load at failure than identical plates with non locking screws. ${ }^{15}$ One of the few studies to test torsional resistance showed three part proximal humeral fracture constructs stabilized with an LCP proximal humeral plate had higher initial torsional stiffness, were more resistant to cyclic torque (four of six pairs), or had decreased maximum rotation after 10,000 cycles than non-locking angled plates. ${ }^{16}$ Though Walsh et al's comparison of a locking plate to a cloverleaf plate in a proximal humeral gap model is blurred by more obviously different plate geometries, their findings of a greater maximum load to failure with locking plates when loaded in 30 degree glenohumeral abduction may still lend credence to the stability of locking plates. ${ }^{17}$ Another study found increased rigidity of cervical constructs stabilized with either of two locked plates compared with the same plates with non-locking screws or a conventional non-locking unicortical plate both initially and after cyclic testing in extension and flexion. Furthermore, unlike the two locked constructs tested, the rigidity of all unlocked constructs decreased significantly after cyclic loading. ${ }^{18}$

Other reports substantiate biomechanical similarities between locking and conventional plate fixation suggesting that the biological advantages offered by the locking plates make them preferable. When testing locking versus non locking mandibular plates in four point bending, Chiodo et al found no statistical significance between groups in either the force at failure or the mechanism of failure and suggested 
that bone quality and surgical technique have more affect on type and degree of failure than whether the plate has locking characteristics. ${ }^{19}$ Biomechanical similarities (yield and load at failure) were also found by Trease et al. between locking and non-locking Tplates applied in either a dorsal or volar orientation when loaded axially. ${ }^{20} \mathrm{~A}$ recent paper described a comparison of distal fibular fractures stabilized with a locking plate and two distal unicortical screws to ones repaired with conventional plates and three distal unicortical screws. $^{21}$ This group found no difference in initial torsional stiffness, torsional stiffness over 2000 cycles or final torque to failure between the two constructs, thereby concluding that locking plates allow fewer cortices per fragment to be engaged.

\section{D: Initial Clinical Results}

Initial clinical outcomes with and results of testing of locking plates were promising. A retrospective study of 387 individual uses of locking plate technology in human patients revealed no instances of implant failure, a low incidence of infection, and healing that was observed to be similar to that seen with external fixation. ${ }^{22}$ Another group which followed 151 fractures repaired with LCPs for one year lauded the complete absence of implant-related complications and remarked how well suited these plates were for difficult fractures where bone stock was unavailable or unsound. ${ }^{3}$ Another locking plate system, the Less Invasive Stabilization System (LISS), was associated with similarly low infection rates in humeral fractures in people. ${ }^{23}$ The authors of these reports cite the inherent stability of these plates and the preservation of the periosteal blood supply afforded by that stability as reasons for the benefits seen.

A few papers can be found discussing clinical impressions on the use of locking

plates in small animal orthopedics. ${ }^{24-26}$ Keller et al. reported on the use of the Compact 
Unilock 2.0/2.4 system in nine cases that were characterized by long bone fractures, cervical spinal fractures and instabilities, and joint instabilities and luxations. ${ }^{24} T h e$ successful use of the Compact Unilock system to promote ligamentous and articular healing bolstered earlier work using this system for tarsal instability. ${ }^{25}$. Unfortunately, because the Compact Unilock system was designed for human maxillofacial surgery, its size (the 2.4 plate is only $2.5 \mathrm{~mm}$ thick) precludes its use in applications where high strength or resistance to fatigue is required. In contrast, Schwandt et al. described their clinical experience with 3.5 LCPs used to stabilize a comminuted radial fracture and spiral tibial fracture in a young Bernese Mountain Dog. ${ }^{26}$ Stability with fewer screws, decreased disruption of periosteal blood supply and overlying soft tissue, absence of an accurate contouring requirement, and freedom to use monocortical screws when the far cortex was unsafe to approach or unavailable due to comminution or implants were cited as reasons for the excellent outcomes reported. More papers can be found describing an earlier evolution of the LCP, the point contact fixator (PC-Fix). The AO Research Institute reported faster healing and increased resistance to infection in fractures stabilized with a PC-Fix compared to fractures managed with conventional DCPs. The reduced bone-implant contact area, absence of plate induced bone necrosis, and the presence of an uninterrupted circumferential callus found with this internal locking fixation were suggested as the basis for the benefits noted. ${ }^{27,28}$

To further evaluate LCPs for veterinary clinical use, they should be tested on a model that simulates a necessary clinical application, in ways previously unreported, and against what is the current standard. In veterinary medicine, distal humeral metaphyseal fractures occur uncommonly but remain an orthopedic challenge: fragments are often 
comminuted, bone purchase distally is usually not adequate for conventional bridging plates, and normal bone anatomy makes contouring necessary and difficult. ${ }^{29,30}$ The inherent stability afforded by LCPs and their independence from contouring suggest an improvement in internal fixation for this type of fracture. We therefore tested an LCP against a similarly sized LC-DCP on a distal humeral metaphyseal gap model. 


\section{CHAPTER II: A Biomechanical Comparison of 3.5 Locking Compression Plate Fixation to 3.5 Limited Contact Dynamic Compression Plate Fixation in a Canine Cadaveric Distal Humeral Metaphyseal Gap Model}

\section{A: Objectives}

It was our hypothesis that in a canine cadaveric, distal humeral, metaphyseal gap model, constructs stabilized with a 3.5 LCP would be at least as stiff as those plated with a 3.5 LC-DCP when tested monotonically to failure in axial compression and when tested cyclically in axial compression and torsion.

\section{B: Materials and Methods}

\section{Experimental Design}

Thirty pairs of cadaveric canine humeri from medium to large breed dogs were

obtained. Ages of the animals were not available, but the mean weight of the dogs was $21.5 \mathrm{~kg}$ (range $13-28.6 \mathrm{~kg}$, standard deviation $4.5 \mathrm{~kg}$ ). After harvesting the limbs, the stripped bones were radiographed in orthogonal planes to ensure skeletal maturity (closed physes) and absence of pathology. The thirty pairs were randomly grouped into three sets of ten pairs corresponding to the three testing modes (set 1 - monotonic axial compression to failure, set 2 - cyclic axial compression, and set 3 - cyclic torsion) and were then fresh frozen at $-20 \mathrm{C}$. One set at a time was gradually thawed at room temperature over 24 - 36 hours before being plated, potted, and subjected to the respective testing. During plating, one humerus from each pair was randomly assigned to 
be plated with a 7 hole $3.5 \mathrm{~mm}$ stainless steel $\mathrm{LCP}^{1}$; the contralateral humerus was plated with a 7 hole $3.5 \mathrm{~mm}$ stainless steel LC-DCP ${ }^{2}$ The distal plate hole was consistently centered over or just proximal to the medial epicondyle on all constructs. The LC-DCPs were anatomically contoured to the medial/caudomedial aspect of the bone while minimal contouring resulted in a gentle curve placed in the LCPs to maximize bone/plate contact, particularly at the most proximal and distal extents of the plate. The LCPs were not contoured anatomically so as to test this reported benefit of locking plates. ${ }^{7,19,31-33}$ Locking screw drill guides were hand tightened into the necessary plate holes of the LCPs after the plate was positioned to ensure that the $2.8 \mathrm{~mm}$ diameter holes drilled into the bone would be aligned with the plate hole. Self-tapping, cortical $3.5 \mathrm{~mm}$ locking screws were placed through both cortices in the distal two and proximal three LCP holes and were power tightened to $1.5 \mathrm{~N}$-m using the manufacturer supplied torque limiting device per manufacturer's recommendations. ${ }^{34}$ Please see Figure 3. The contralateral humeri of the set were plated with LC-DCPs by drilling $2.5 \mathrm{~mm}$ diameter holes and then inserting self-tapping, non-locking, $3.5 \mathrm{~mm}$ cortical screws with lengths sufficient to engage the trans cortex. Screws in the proximal three and distal two plate holes were driven initially under power, then hand tightened by one of two investigators (DF/OL) as if in a clinical setting. ${ }^{35}$ A $20 \mathrm{~mm}$ ostectomy centered under the third and fourth most distal screw holes and a transverse subcapital osteotomy were performed on all humeri. Particular attention was paid to ensure that the saw blade never contacted the plate. The proximal aspect of all pairs was then potted in custom jigs with polymethylmethacrylate ${ }^{3}$

\footnotetext{
${ }^{1}$ Synthes (USA), Paoli, PA, USA.

${ }^{2}$ Synthes (USA), Paoli, PA, USA.

${ }^{3}$ COE Fast Set Tray Plastic, GC America, Chicago, IL
} 
(PMMA) so that the longitudinal axes of the plates were perpendicular to the horizontal. For each set slated for axial compression tests (monotonic to failure, set 1 and cyclic to 10,000 cycles, set 2 ), humeri were individually bolted via their proximal jigs to a servohydraulic loading system ${ }^{4}$ and lowered into a setting disc of PMMA until the distal aspect of the medial portion of the condyle made contact with the machine base. A mold of PMMA was then created of the distal condyle to allow equal load distribution during testing. Special attention was given to ensure that no PMMA made any contact with either the plate or the screws of any construct and that the plate would not make contact with the base if displacement occurred. Please see Figure 4. For the last test, the distal aspects of the remaining ten pairs of constructs were potted in custom jigs similar to those on the proximal aspects of the constructs in such a way that the bases of the proximal and distal jigs were parallel and aligned. The condyles were centered and stabilized within these jigs by means of PMMA and two screws placed orthogonally to each other in the distal jigs. The medial aspect of the condyles of these constructs had no contact with either the cement or any part of the jig. Specimens of each set were wrapped in saline-soaked gauze while awaiting testing.

\section{Mechanical Testing}

Axial compression tests were performed on a servohydraulic material testing machine ${ }^{c}$ in load control. The first ten pairs of humeri were tested in axial compression until catastrophic failure; failure was defined as the first point of major discontinuity in the load versus displacement curve caused by bone fracture or implant failure. After an initial preload of $0.4 \mathrm{~kg}$ was applied, compression was initiated at $5 \mathrm{~mm} / \mathrm{min}$ with data

\footnotetext{
${ }^{4}$ MiniBionix 858, MTS Corporation, Eden Prairie, MN
} 
capture set at $10 \mathrm{~Hz}$. Load versus deformation curves were generated. Stiffness, defined as load per displacement in the elastic region of the curves, and ultimate strength, defined as the load at which additional deformation did not require additional load, were evaluated. Qualitative descriptions at failure were noted. For the second ten pairs, the load was set to vary between 20 and $1470 \mathrm{~N}$ sinusoidally at a frequency of $2 \mathrm{~Hz}$ for 10,000 cycles. These inputs were a result of pre-testing experiments performed on one sample pair. Maximum and minimum absolute positions and force data were captured for every cycle. In addition, load deformation curves were captured every 3000 cycles. Total subsidence, deformation per cycle, and stiffness per cycle were evaluated. The number of revolutions needed to tighten locking screws to $1.5 \mathrm{Nm}$ and conventional screws to subjective pre-testing tightness (if possible) was recorded. The last ten pairs of humeri were fastened to a torsional servohydraulic testing frame ${ }^{5}$ through the proximal and distal jigs. This machine was set in displacement control to cycle at $0.5 \mathrm{~Hz}$ with an amplitude of $+/-15$ degrees, as the strength of the feedback signal and the control settings of the actuator at these low loads precluded the use of this machine under load control. Torque versus angular deformation data were collected at $100 \mathrm{~Hz}$ over 500 cycles, and the resulting torsional stiffness versus cycle curves were evaluated. As in the prior dynamic test, pre-testing experiments performed on one sample pair guided the choice of these values. Torsional stiffness was defined as the slope of the elastic portion of the torque versus angle curve. Qualitative assessments of the constructs post testing were compared and included screw tightness, and mode of construct failure if applicable.

\footnotetext{
${ }^{5}$ Instron 8850, Norwood, MA
} 


\section{Statistical Analysis}

An initial power analysis was performed based on torsional data previously

reported for $3.5 \mathrm{LCPs} .{ }^{10}$ It was found that nine pairs of cadaveric bones would be needed per test to able to detect a 200degree/m mean paired difference in twist with a power of $82 \%$.

For static and cyclic axial loading, differences between each pair of humeri (one assigned to the LCP grouping and the other to the LC-DCP grouping) were computed, and normal probability plots generated to check if the data had a Gaussian distribution. Subsequently, a paired mean difference (with a $95 \%$ confidence interval) was computed, and a paired t-test applied to test the null hypothesis that the LCP constructs were not significantly different from the LC-DCP constructs. Torsion testing data were modeled using mixed models analysis of variance with plate type and cycle (every $20^{\text {th }}$ cycle up to a maximum of 500) as fixed effects and pair of humeri as the random effect. The level of significance was set at alpha $=0.05$. All analyses were performed using SAS (version 9.1.3 service pack 4, Cary, NC).

\section{C: Results}

\section{Static Axial Compression}

When loaded in axial compression up to 2000 Newtons, humeral constructs stabilized with an LCP were significantly stiffer than those plated with LC-DCPs ( $\mathrm{P}=0.0004$ ). Mean stiffness of the LCP constructs was 993 (SD 343) N/mm compared with 447 (SD 61.7) N/mm for the LC-DCP constructs. Ultimate strengths of the two constructs were not significantly different $(\mathrm{p}=0.1960)$. Mean ultimate strength of the 
LCP constructs was 4116 (SD 749) N and was 4406 (SD 557) $\mathrm{N}$ for the LC-DCP constructs.

No consistent mode of failure was noted in the LCP constructs. Four LCPs failed by bending, four LCP constructs failed when the proximal screws cut through the proximal bone fragment, and two LCP constructs failed when the distal screws cut through the distal bone fragment. Eight LC-DCP constructs failed by subsidence of the distal fragments likely primarily from pull out of the distal screws, one construct failed by severe bending of distal screws, and the last construct failed by bending of the proximal screws. All locking screws appeared fully seated prior to testing.

\section{Cyclic Axial Compression}

When cyclically loaded in axial compression, the mean stiffness of the LCP constructs over the course of testing was significantly less than that of constructs stabilized with an LC-DCP $(\mathrm{P}=0.0029)$. The mean stiffness of the LCP constructs was 2752 (SD 320) N/mm, and the mean stiffness of the LC-DCP constructs was 4342 (SD 1136) $\mathrm{N} / \mathrm{mm}$. The LCP constructs experienced significantly more average displacement during each loading cycle $(0.507 \mathrm{SD} 0.058 \mathrm{~mm})$ than the LC-DCP constructs $(0.344 \mathrm{SD}$ $0.07 \mathrm{~mm})(\mathrm{P}=0.0002)$. However, total displacement was not significantly different between groups $(\mathrm{P}=0.084)$ as the LCP constructs subsided $0.61(\mathrm{SD} 0.19) \mathrm{mm}$ and the LC-DCP constructs subsided 0.85 (SD 0.45) $\mathrm{mm}$.

The proximal of the distal two screws was loosened in all constructs stabilized with LCPs, and in those constructs where additional screw loosening occurred, the proximal of the distal screws required more revolutions to re-lock than the other loosened 
screws. Movement was consistently identified in the locking constructs as elastic bending in the visible portion of the distal screws between the plate and bone. One construct appeared to have an incompletely seated locking screw (two threads showing) at the proximal position of the distal fragment prior to testing. An additional attempt was made to seat this screw fully prior to testing, but the torque governor restricted further rotation. There were no observable indications that this screw was not fully locked. No significant difference in outcomes was appreciated by excluding this data, and as proper protocol had been followed during the plating and potting procedures for this sample, it remained in the set. Careful visual inspection of the remaining LCP screw heads of this set revealed three other constructs, each with one screw (the most distal of the proximal fragment in one and the most proximal of the distal fragment in two), where a portion of one thread was still visible. As above, the torque governor prevented any further tightening of these screws and all indications pointed to these screws being fully seated and locked.

Movement in the LC-DCP constructs was consistently noted as elastic deformation of the plate at the gap. In those constructs that experienced screw loosening $(8 / 10)$, the proximal of the distal screws was loose in $75 \%(6 / 8)$ of the constructs and appeared looser than other screws when additional screw loosening was present. Screw loosening occurred more in the most distal of the proximal screws in one construct and in the most distal screw in the last construct compared to other screws in those constructs. The remaining two constructs showed no screw loosening.

\section{Cyclic Torsion}

Constructs stabilized with LC-DCPs showed significantly greater resistance to torsion over 500 cycles than those stabilized with LCPs $(\mathrm{P}<0.0001)$. Please see Figure 6. 
The average torsional stiffness of the LC-DCPs was 0.2286 (SE 0.028 ) N/mm and of the LCPs over 500 cycles was $0.1872(0.0281) \mathrm{N} / \mathrm{mm}$. However, a difference in resistance to torsional loading was not significant during the first 280 cycles $(\mathrm{P}=0.096)$ when the LCDCP constructs exhibited an average torsional stiffness of 0.2774 (SE 0.039$) \mathrm{N} / \mathrm{mm}$ compared to that of the LCPs, $0.2548(0.039) \mathrm{N} / \mathrm{mm}$.

Similar to the dynamic axial testing, one screw in one of the torsion constructs appeared incompletely seated by two threads, and two additional screws in two more constructs subjectively appeared not to be fully seated with at most one thread visible above the surface of the LCP. All three screws were positioned in the proximal hole of the distal fragment. Further tightening of all of these screws was prevented by the torque limiter. Exclusion of the construct in which two threads were visible made no difference to our results, so it remained for subsequent calculations. Exclusion of all three constructs would have severely limited the power of our analysis, and as our application protocol had not differed during the plating of these constructs, they remained in the analysis. All constructs exhibited screw loosening, screw breakage, or both. Five LCP constructs exhibited screw breakage with four of these failures occurring in the distal fragment (the distal screw alone in two, the proximal screw in one, and both screws in one) and one occurring in the proximal fragment when both distal screws failed. The bone cracked proximally in two LCP constructs. The remaining three LCP constructs exhibited screw loosening only with at least one of the distal screws requiring at least one revolution post-testing to achieve $1.5 \mathrm{Nm}$. The proximal screws in these three constructs were tightened less than one quarter of a revolution before reaching $1.5 \mathrm{Nm}$. Interestingly, the screw that appeared incompletely seated by two threads exhibited no loosening. 
Five LC-DCP constructs had one screw break each; three of these were the distal screw in the proximal fragment, and the remaining two occurred in the distal fragment (one proximally, one distally). Three LC-DCP constructs required more than one turn of the distal fragment screws to tighten whereas the other two LC-DCP constructs required more than one turn of at least one of the proximal fragment screws to tighten and the distal fragment screws were less than a quarter turn loose.

\section{D: Discussion}

It was our hypothesis that in a comminuted metaphyseal fracture model, constructs stabilized with LCPs would be as stiff as those stabilized with conventional LC-DCPs when subjected to static axial and dynamic axial and torsional loads. Though it proved valid in monotonic loading to failure, our hypothesis was not substantiated in the two dynamic tests, as constructs stabilized with the LC-DCPs demonstrated significantly greater stiffness than their locked counterparts over the course and at the end of all cyclic testing.

\section{Static Load to Failure}

Results of our static axial compression tests supported the hypothesis that LCPs provide greater stiffness than similar non-locking plates. This is in agreement with recent work in which the only notable difference from our protocol was the use of a double plating arrangement. ${ }^{12}$ The reason for this rigidity can be attributed to the interface between the screw head and locking plate that transfers axial loads through the screws to the bone. In comparison, once the frictional force at the plate-bone interface in nonlocking plates has been overcome, a shear force is created at the screw-bone interfaces as 
the screws begin to toggle. As bone is better able to resist compressive stress than shear stress, the greater stiffness of locking plates may be explained. ${ }^{4}$

The absolute load at failure and the manner of failure must be borne in mind when considering the finding of similar ultimate strengths of the LCP and LC-DCP constructs in axial compression. The force necessary to cause failure in this model was roughly 2628 times the average body weight of the dogs whose humeri were used in this study. The clinical significance of a construct reaching failure at such a supra-physiologic force is unknown. Secondly, of the nine locking constructs that experienced catastrophic failure, $66 \%$ failed precipitously by cut through of the bone with continued angular stability of those screws which experienced the cut through, while the remaining three constructs failed by gap closure secondary to plate deformation. In comparison, $90 \%$ of the nonlocking constructs failed by gradual plate subsidence subsequent to screw pull-out and bending, and possibly plate bending. Gap closure was greater than $10 \mathrm{~mm}$ in all of these constructs. These findings indicate that prior to failure (which occurred at supraphysiological levels), locking constructs maintained their rigidity. On the other hand, the LC-DCP constructs experienced near continuous deformation at sub-failure load levels. Clinically, this may translate to a greater risk of delayed or non-union with the LC-DCPs due to movement at the fracture gap.

\section{Cyclic Loading}

Results of our dynamic testing showed that constructs stabilized with the LCDCPs were stiffer in cyclic axial compression and cyclic torsion than those stabilized with the LCPs. Though dissimilar results have been reported for cyclic torsional ${ }^{11,16,21}$ and cyclic compression ${ }^{13}$ testing, the only group to have used a small animal fracture gap 
model reported similar findings from their torsion testing ${ }^{10}$. Different loading applications, experimental set-ups, implant types, construct materials, and fracture models make direct comparisons between our work and that cited difficult.

The authors' decision not to contour the 3.5 LCPs anatomically resulted in a plate-bone offset and less screw purchase in the distal fragment. We believe these factors, coupled with a small number of possibly partially seated locking screws, likely contributed to the locking constructs inferior stiffness during the cyclic testing.

\section{Offset}

The decision to forego anatomic contouring of the LCPs was based on recommendations to maintain an offset ${ }^{5,7}$, on other work which allowed an offset provided soft tissue closure wasn't compromised ${ }^{33}$, and on reports which specifically looked at the affects of offset and concluded that a small offset was allowable. ${ }^{13,31,36}$ Therefore, the LCPs were contoured so the most distal and proximal screw holes lay adjacent to the bone, but no effort was made to ensure that the remainder of the plate contacted the bone. This led to at least a minimally apparent offset in all locking constructs, most noticeable under the second most distal screw hole. Please see Figure 7.

Two main explanations are offered as to why this offset may have resulted in the decreased stiffness observed in the locking constructs: a lower area moment of inertia and the combination of decreased support of the implant by the bone coupled with an increased moment from a larger lever arm. Just as the stiffness of external fixators has been shown to be inversely proportional to the distance between bone and connecting bar, increasing offset may decrease the area moment of inertia of the construct and result in a

decreased resistance to loading. ${ }^{37}$ Secondly, any offset introduced between the plate and 
bone will cause any force applied through the plate to act through the moment arm of the exposed screw and result in a moment or torque about the cis cortex. ${ }^{36}$ The addition of this force coincides with the loss of the buttressing effect of the bone on the portion of the screws within the offset and the underside of the plate. Because of the increased levering force and without a supporting reactive force, the unsupported portion of the shank of the screws, the threads at the screw-plate interface, and the offset plate itself experience more stress and are susceptible to greater deformation than if no offset were present. Please see Figure 8. Cyclic deformation could then lead to screw loosening/unlocking, particularly at the threads of the plate hole, which are more prone to deformation than if they completely encircled the screw head. Greatest screw loosening would then be expected where the greatest cyclic displacement occurred. This was seen in our data as the proximal of the distal screws, where the offset was consistently greatest, was the screw most often and most severely loosened.

The LCPs in our study were contoured to allow an offset, but due to the metaphyseal nature of our constructs and to more closely simulate the clinical scenario, the offset was not rigidly controlled. Based on subjective estimates by all four researchers, it is likely that the greatest offset of each construct measured around two millimetres, but was unlikely to have ever exceeded $0.5 \mathrm{~cm}$. Since our testing, Ahmad et al. have found that in a diaphyseal gap model, no difference could be appreciated between constructs stabilized with 4.5 LCPs or 4.5 DCPs when subjected to static and cyclic axial compression and torsional testing provided that the offset was not greater than two millimetres, and that structural stability of these constructs suffered significantly at an offset of five millimetres. ${ }^{38}$ A limitation of our study then is the uncertainty 
regarding the amount of offset under each screw hole or total amount of offset. Though this could be quantified in subsequent studies, the effect of offset on a curved or contoured plate has yet to be investigated.

As a result of the results of our dynamic testing, we suggest contouring all locking plates so as to eliminate any offset. Though it is known that compression of the periosteum causes early temporary porosity that can lead to increased rates of infection, the minimal amount of periosteal pressure that will result in significant vascular impairment is unknown. Ideally, the biological benefits of locking plates would not be diminished by compression-free contact with bone that would occur after appropriate contouring.

\section{Decreased screw purchase}

Though the design of the combination hole of LCPs is such that deformation should occur first through the compression portion of the hole (and not the threaded portion), ${ }^{8}$ concerns that contouring could deform the threaded portion of the plate leant additional support to the decision not to contour the plates to anatomically conform along the longitudinal axis of the distal humeri. ${ }^{39}$ Without having contoured a twist into the locking plates in this study, the second most distal screw in $95 \%$ of our constructs did not reach the trans metaphyseal cortex and only engaged the medial epicondylar crest, albeit both cortices of this crest. Please see Figure 7. Unlike screws in the LC-DCP constructs that could be angled up to 14 degrees in the transverse and 80 degrees in the longitudinal planes, locking screws can only be placed perpendicular through the plate, or no more than five degrees from the perpendicular, to avoid cross-threading and a significant reduction in construct stability. ${ }^{32,34}$ This inability of the surgeon to alter the angle of the 
screw within the hole and still achieve a locked screw is a common complaint of current locking plate design. ${ }^{12,26,39-41}$ Comparative stiffness during cyclic testing was likely adversely affected because of this, but the contribution from decreased bone purchase, particularly significant in the metaphysis where the cortices are thinner and less dense $^{12,39}$, or from purchase on only one side of the bone's neutral axis is difficult to ascertain. If possible, plate adaptation to allow increased screw purchase should be attempted.

\section{Possible incomplete seating}

Insufficient plate adaptation may not have been the only major cause of the decreased stiffness exhibited by the locking constructs during cyclic testing; in at least two but possibly up to seven of these constructs (three in compression and four in torsion), at least one screw in each plate did not appear to be seated fully and had some portion of a thread visible above the plate, despite being seated and double checked with the use of the torque limiter as per manufacturer's recommendations. Please see Figure 9. Incomplete screw seating could occur if during screw insertion, greater than $1.5 \mathrm{Nm}$ of resistance was encountered prior to the complete engagement of all threads on the screw head; the torque limit of $1.5 \mathrm{Nm}$ is the manufacturer's recommended limit for $3.5 \mathrm{~mm}$ screws and has been set to avoid cold welding and the subsequent difficulties encountered in ex-plantation in human patients. ${ }^{3,23,34}$ This resistance could occur if debris clogged the cutting flutes of the self-tapping screws, if the screw, under power, taps a hole divergent from the aligned, pre-drilled one, or if the drill guide is inadvertently misthreaded. Despite our diligence in keeping the taps clean between specimens and our attention to detail when powering the screws or threading the guides, the above situations 
may still have occurred and are a reported complication with locking plates. ${ }^{42}$ An incompletely seated locking screw will either not effectively lock and create angular stability or will be at increased risk of loosening and losing axial and angular stability. This is especially true with the LCP system as the conically shaped locking head only creates a locked interface when the largest diameter threads on the screw head, in other words the last screw head threads to interdigitate with the plate threads, fully engage the plate. Please see Figures $1 \& 2$. The authors believe that in the rare instance that a screw does not appear completely seated, tightening beyond the $1.5 \mathrm{Nm}$ is indicated as the risk of screw loosening and implant failure far outweighs the concerns over cold-welding (which usually only occurs with more ductile metals like titanium) in the event of explantation.

\section{E: Conclusions}

In conclusion, it is important to note this study should not be interpreted as a condemnation of locking technology or LCPs; the biomechanical data reported is likely more a reflection of incorrect application or possibly use in incorrect applications than of actual implant inferiority. These plates were developed in response to the paradigm shift from rigid to appropriately flexible fixation characteristic of biological osteosynthesis. The new emphasis on vascular preservation and minimally invasive methods required an implant with inherent stability that had previously been achieved by bone-implant compression. The fixed angle interface provided this stability. Locking technology then was not designed to be more stable than conventional plates. Instead, it was designed to incorporate and be used with the principles of biological osteosynthesis to promote 
healing and to address the risk of infection that was partially attributable to the periosteal vascular impairment seen with compression plates. Tepic and others reported success in this when they showed unequivocally early, solid healing and increased resistance to infection with the use of the PC-Fix, an early generation locking plate on which the LCP was modelled. ${ }^{2,27,28}$ As angle stable fixation evolved alongside methods of biological osteosynthesis, additional benefits of locking technology began to be realized. The human literature filled with reports of clinical successes, and the veterinary literature is beginning to follow suite. ${ }^{24-26}$.

It is left to orthopaedists to exploit the benefits of this new technology but to avoid the temptation to overestimate its benefits and uses. We are not the first to admit that deficiencies in biomechanical testing or in clinical results, borne from such overestimation of locking technology, are more likely from improper technique or use than any actual deficiency in the implant itself. ${ }^{3,43-45}$ We conclude from our static compression testing that the use of 3.5 LCPs in severely comminuted, distal humeral, fractures may be indicated. Furthermore, we suggest that LCPs should only be used in these types of fractures if screw tightness is governed by visual evaluation and if these plates are appropriately contoured. The LCP will be a useful addition to the orthopaedists armamentarium but, like everything else, only when correctly applied in the appropriate circumstance. 


\section{REFERENCES}

1. Conzemius M, Swainson S: Fracture fixation with screws and bone plates. Veterinary Clinics of North America-Small Animal Practice 29:1117-+, 1999.

2. Perren SM: Evolution of the internal fixation of long bone fractures. The scientific basis of biological internal fixation: choosing a new balance between stability and biology. J Bone Joint Surg Br 84:1093-1110, 2002.

3. Sommer C, Gautier E, Muller M, et al: First clinical results of the Locking Compression Plate (LCP). Injury 34 Suppl 2:B43-54, 2003.

4. Egol KA, Kubiak EN, Fulkerson E, et al: Biomechanics of locked plates and screws. Journal of Orthopaedic Trauma 18:488-493, 2004.

5. Wagner M: General principles for the clinical use of the LCP. Injury 34 Suppl 2:B3142, 2003.

6. Perren SM: Backgrounds of the technology of internal fixators. Injury 34 Suppl 2:B1$3,2003$.

7. Frigg R: Locking Compression Plate (LCP). An osteosynthesis plate based on the Dynamic Compression Plate and the Point Contact Fixator (PC-Fix). Injury 32 Suppl 2:63-66, 2001.

8. Frigg R: Development of the Locking Compression Plate. Injury 34 Suppl 2:B6-10, 2003.

9. Florin M, Arzdorf M, Linke B, et al: Assessment of stiffness and strength of 4 different implants available for equine fracture treatment: a study on a 20 degrees oblique long-bone fracture model using a bone substitute. Vet Surg 34:231-238, 2005.

10. Aguila AZ, Manos JM, Orlansky AS, et al: In vitro biomechanical comparison of limited contact dynamic compression plate and locking compression plate. Veterinary and Comparative Orthopaedics and Traumatology 18:220-226, 2005.

11. Gardner MJ, Brophy RH, Campbell D, et al: The mechanical behavior of locking compression plates compared with dynamic compression plates in a cadaver radius model. J Orthop Trauma 19:597-603, 2005. 
12. Korner J, Diederichs G, Arzdorf M, et al: A biomechanical evaluation of methods of distal humerus fracture fixation using locking compression plates versus conventional reconstruction plates. J Orthop Trauma 18:286-293, 2004.

13. Fulkerson E, Egol KA, Kubiak EN, et al: Fixation of diaphyseal fractures with a segmental defect: A biomechanical comparison of locked and conventional plating techniques. Journal of Trauma-Injury Infection and Critical Care 60:830$835,2006$.

14. Seide K, Triebe J, Faschingbauer M, et al: Locked vs. unlocked plate osteosynthesis of the proximal humerus - a biomechanical study. Clin Biomech (Bristol, Avon) 22:176-182, 2007.

15. Stoffel K, Booth G, Rohrl SM, et al: A comparison of conventional versus locking plates in intraarticular calcaneus fractures: a biomechanical study in human cadavers. Clin Biomech (Bristol, Avon) 22:100-105, 2007.

16. Weinstein DM, Bratton DR, Ciccone WJ, 2nd, et al: Locking plates improve torsional resistance in the stabilization of three-part proximal humeral fractures. $\mathrm{J}$ Shoulder Elbow Surg 15:239-243, 2006.

17. Walsh S, Reindl R, Harvey E, et al: Biomechanical comparison of a unique locking plate versus a standard plate for internal fixation of proximal humerus fractures in a cadaveric model. Clin Biomech (Bristol, Avon) 21:1027-1031, 2006.

18. Spivak JM, Chen D, Kummer FJ: The effect of locking fixation screws on the stability of anterior cervical plating. Spine 24:334-338, 1999.

19. Chiodo TA, Ziccardi VB, Janal M, et al: Failure strength of 2.0 locking versus 2.0 conventional Synthes mandibular plates: A laboratory model. J Oral Maxillofac Surg 64:1475-1479, 2006.

20. Trease C, McIff T, Toby EB: Locking versus nonlocking T-plates for dorsal and volar fixation of dorsally comminuted distal radius fractures: a biomechanical study. J Hand Surg [Am] 30:756-763, 2005.

21. Kim T, Ayturk UM, Haskell A, et al: Fixation of osteoporotic distal fibula fractures: A biomechanical comparison of locking versus conventional plates. J Foot Ankle Surg 46:2-6, 2007. 
22. Haas N, Hauke C, Schutz M, et al: Treatment of diaphyseal fractures of the forearm using the Point Contact Fixator (PC-Fix): Results of 387 fractures of a prospective multicentric study (PC-Fix II). Injury-International Journal of the Care of the Injured 32:Sb51-Sb62, 2001.

23. Hertel R, Eijer H, Meisser A, et al: Biomechanical and biological considerations relating to the clinical use of the Point Contact-Fixator--evaluation of the device handling test in the treatment of diaphyseal fractures of the radius and/or ulna. Injury 32 Suppl 2:B10-14, 2001.

24. Keller MA, Voss K, Montavon PM: The ComPact UniLock 2.0/2.4 system and its clinical application in small animal orthopedic. Veterinary and Comparative Orthopaedics and Traumatology 18:83-93, 2005.

25. Voss K, Keller M, Montavon PM: Internal splinting of dorsal intertarsal and tarsometatarsal instabilities in dogs and cats with the ComPact Unilock 2.0/2.4 (TM) system. Veterinary and Comparative Orthopaedics and Traumatology 17:125-130, 2004.

26. Schwandt CS, Montavon PM: Locking Compression Plate fixation of radial and tibial fractures in a young dog. Veterinary and Comparative Orthopaedics and Traumatology 18:194-198, 2005.

27. Arens S, Eijer H, Schlegel U, et al: Influence of the design for fixation implants on local infection: experimental study of dynamic compression plates versus point contact fixators in rabbits. J Orthop Trauma 13:470-476, 1999.

28. Tepic S, Remiger AR, Morikawa K, et al: Strength recovery in fractured sheep tibia treated with a plate or an internal fixator: an experimental study with a two-year follow-up. J Orthop Trauma 11:14-23, 1997.

29. Vannini R, Olmstead ML, Smeak DD: An Epidemiological-Study of 151 Distal Humeral Fractures in Dogs and Cats. Journal of the American Animal Hospital Association 24:531-536, 1988.

30. Vannini R, Smeak DD, Olmstead ML: Evaluation of Surgical Repair of 135 Distal Humeral Fractures in Dogs and Cats. Journal of the American Animal Hospital Association 24:537-545, 1988. 
31. Haug RH, Street CC, Goltz M: Does plate adaptation affect stability? A biomechanical comparison of locking and nonlocking plates. J Oral Maxillofac Surg 60:1319-1326, 2002.

32. Kaab MJ, Frenk A, Schmeling A, et al: Locked internal fixator - Sensitivity of screw/plate stability to the correct insertion angle of the screw. Journal of Orthopaedic Trauma 18:483-487, 2004.

33. Korner J, Lill H, Muller LP, et al: The LCP-concept in the operative treatment of distal humerus fractures--biological, biomechanical and surgical aspects. Injury 34 Suppl 2:B20-30, 2003.

34. Small Fragment Locking Compression Plate (LCP) Technique Guide - Synthes USA, in, Vol Synthes USA, 2003.

35. Johnson AL, Houlton, J.E.F., Vannini, R.: AO Principles of Fracture Management in the Dog and Cat. Davos, CH AO Publishing, 2005.

36. Kowalski MJ, Schemitsch EH, Harrington RM, et al: A comparative biomechanical evaluation of a noncontacting plate and currently used devices for tibial fixation. $\mathrm{J}$ Trauma 40:5-9, 1996.

37. Bouvy BM, Markel MD, Chelikani S, et al: Ex vivo biomechanics of KirschnerEhmer external skeletal fixation applied to canine tibiae. Vet Surg 22:194-207, 1993.

38. Ahmad M, Nanda R, Bajwa AS, et al: Biomechanical testing of the locking compression plate: when does the distance between bone and implant significantly reduce construct stability? Injury 38:358-364, 2007.

39. Haidukewych GJ: Innovations in locking plate technology. J Am Acad Orthop Surg 12:205-212, 2004.

40. Gardner MJ, Helfet DL, Lorich DG: Has locked plating completely replaced conventional plating? Am J Orthop 33:439-446, 2004.

41. Phisitkul P, McKinley TO, Nepola JV, et al: Complications of locking plate fixation in complex proximal tibia injuries. J Orthop Trauma 21:83-91, 2007.

42. Charalambous CP, Siddique I, Valluripalli K, et al: Proximal humeral internal locking system (PHILOS) for the treatment of proximal humeral fractures. Arch Orthop Trauma Surg 127:205-210, 2007. 
43. Namazi H, Mozaffarian K: Awful considerations with LCP instrumentation: a new pitfall. Arch Orthop Trauma Surg 127:573-575, 2007.

44. Zura RD, Browne JA: Current concepts in locked plating. J Surg Orthop Adv 15:173-176, 2006.

45. Hernanz Gonzalez Y, Diaz Martin A, Jara Sanchez F, et al: Early results with the new internal fixator systems LCP and LISS: a prospective study. Acta Orthop Belg 73:60-69, 2007. 


\section{APPENDIX 1: FIGURES}

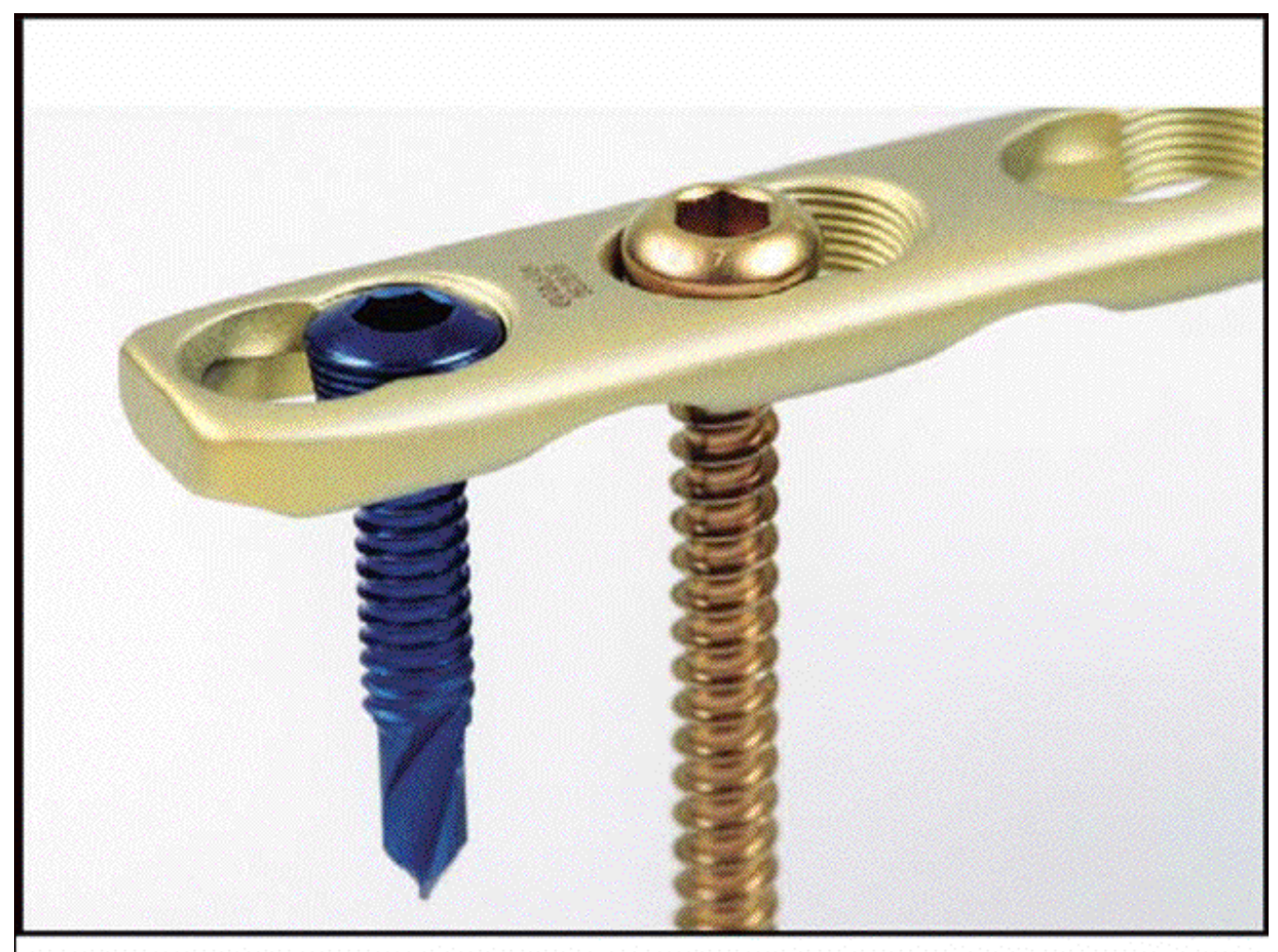

Figure 1: Locking compression plate (LCP). Note the locking screw on the left and conventional screw on the right. 


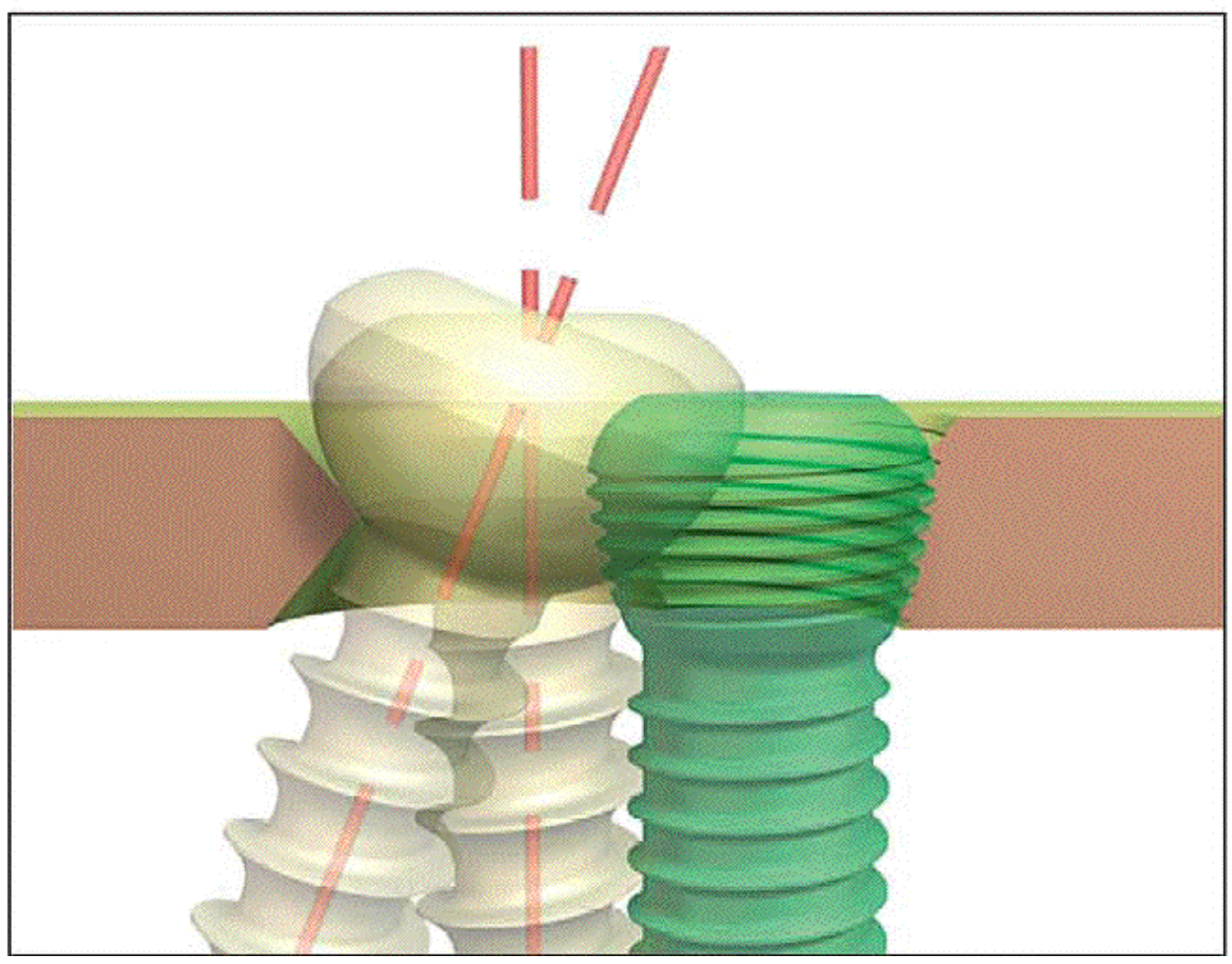

Figure 2: Dynamic Compression Unit (DCU) of the LCP. The non-threaded section on the left accepts conventional, non-locking screws, and the threaded section on the right accepts locking screws. 


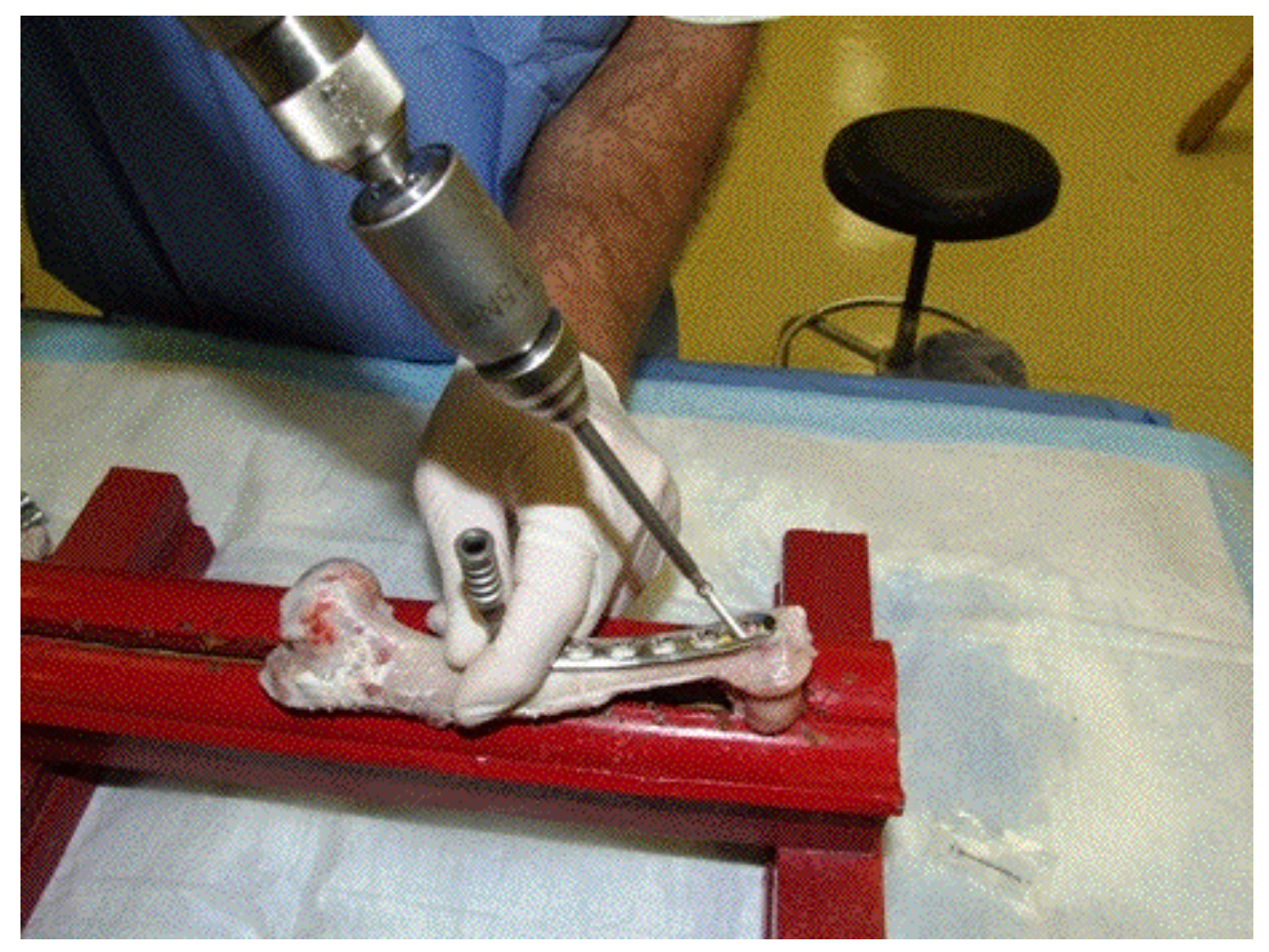

Figure 3: Application of the LCP. Note the threaded drill guide screwed into the proximal aspect of the plate and the $1.5 \mathrm{Nm}$ torque limiter being used during screw tightening. 


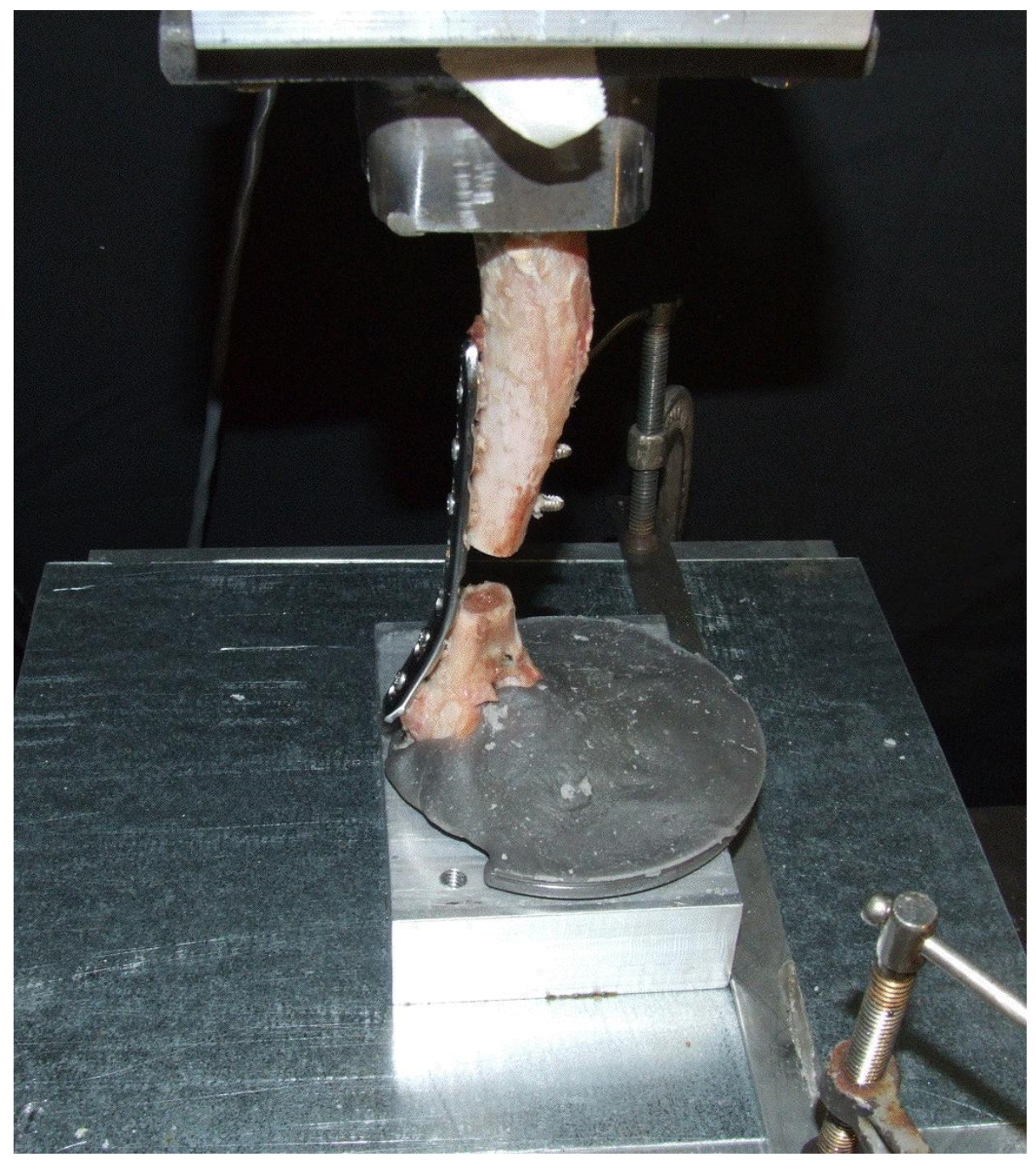

Figure 4: Representative LC-DCP construct during axial testing. 
Load vs Deformation - Humeral Pair 3B

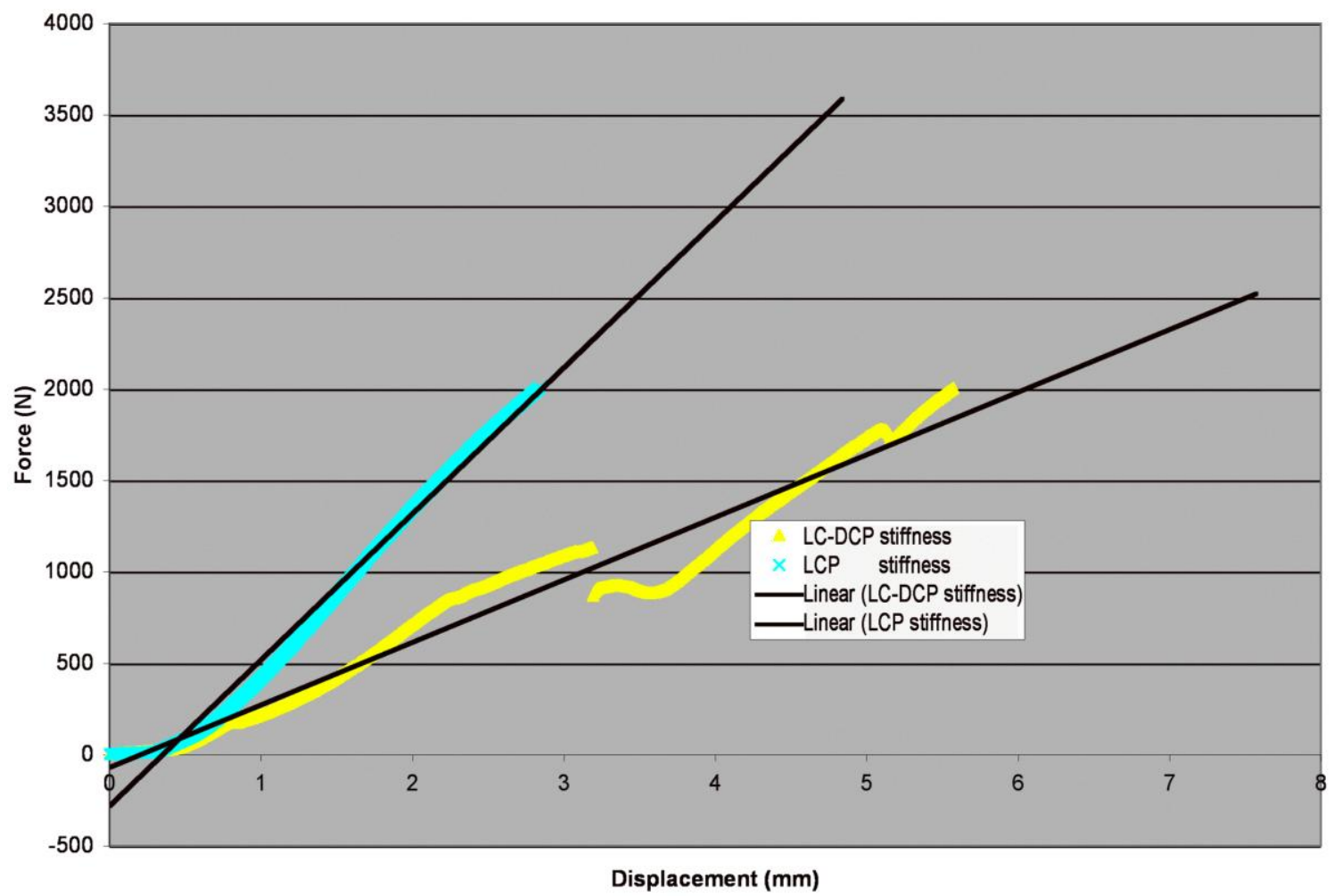

Figure 5: Load vs. Deformation curve of representative humeral pair loaded monotonically in axial compression. 


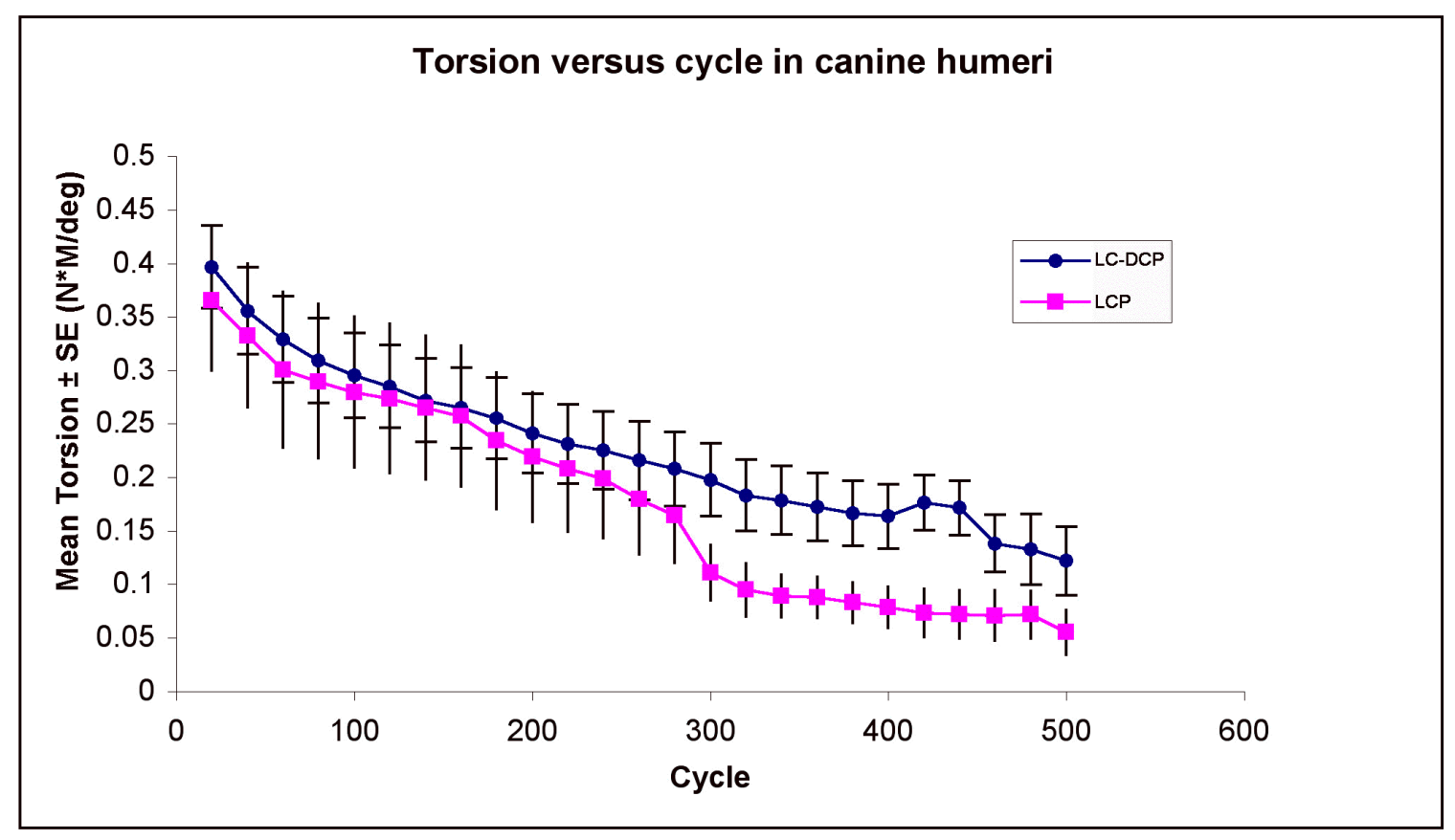

Figure 6: Mean resistance to torsional loading over 500 cycles. 


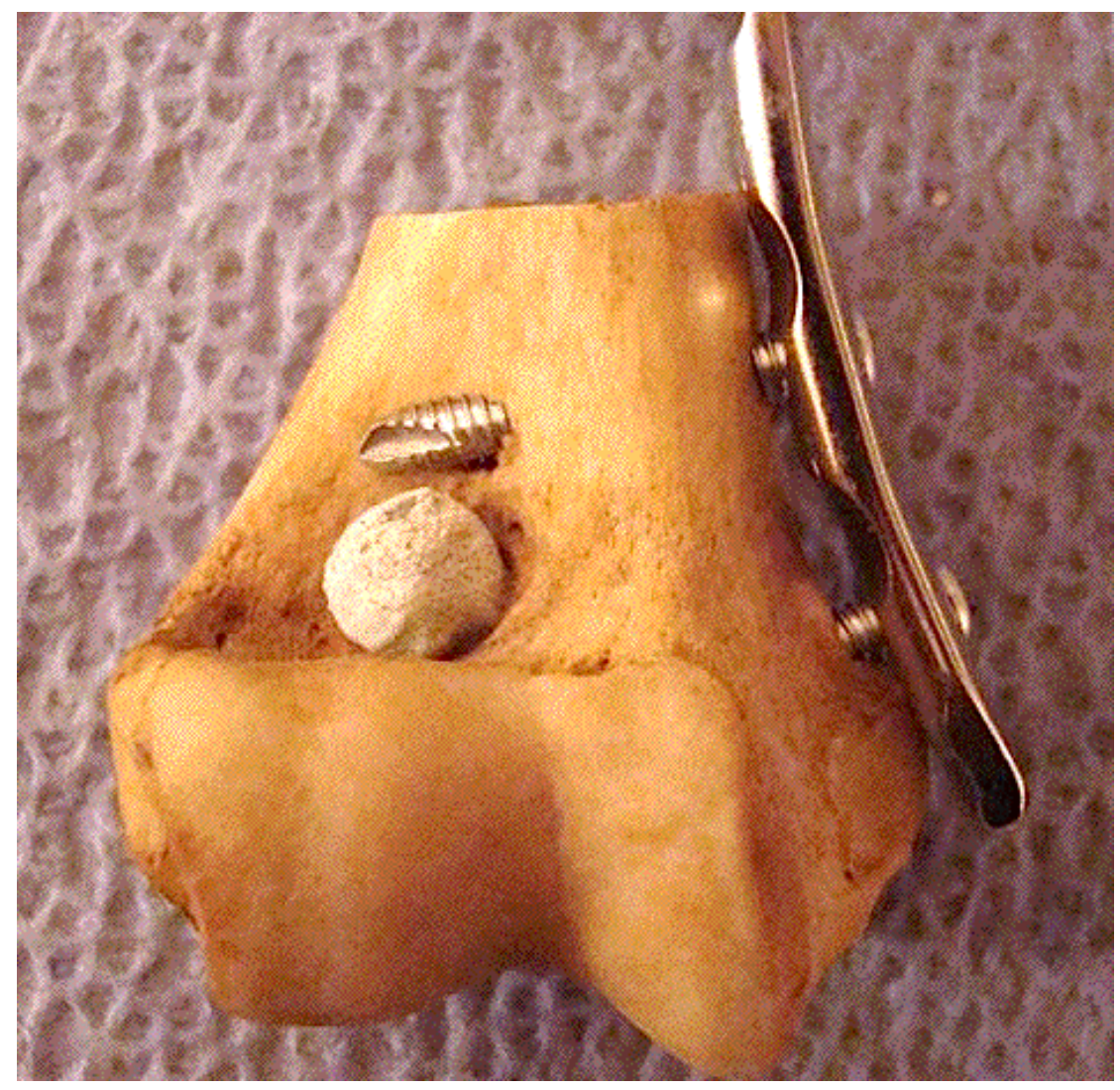

Figure 7: Distal portion of LCP construct after testing. Note the offset present between the plate and bone between the two screws. Note also how the proximal screw does not penetrate the trans cortex. 


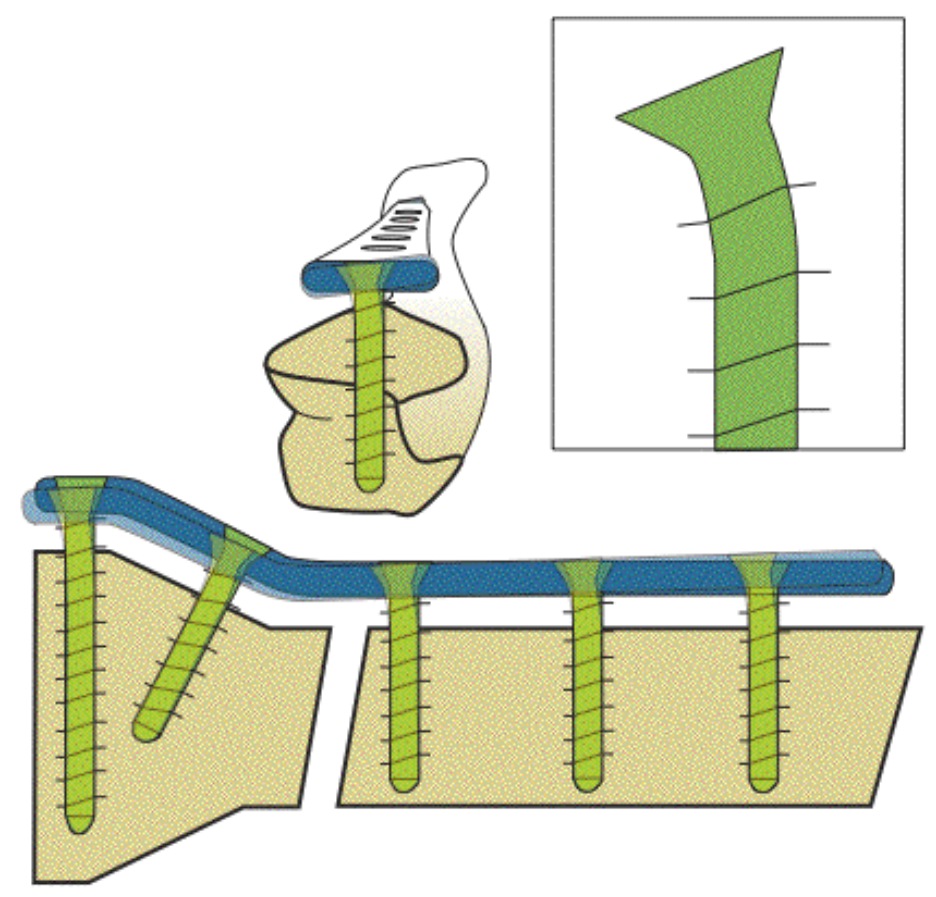

Figure 8: Illustration depicting an exaggeration of the loss of the buttressing of the bone on the underside of the plate and the exposed portions of the screws secondary to a contiguous offset. 


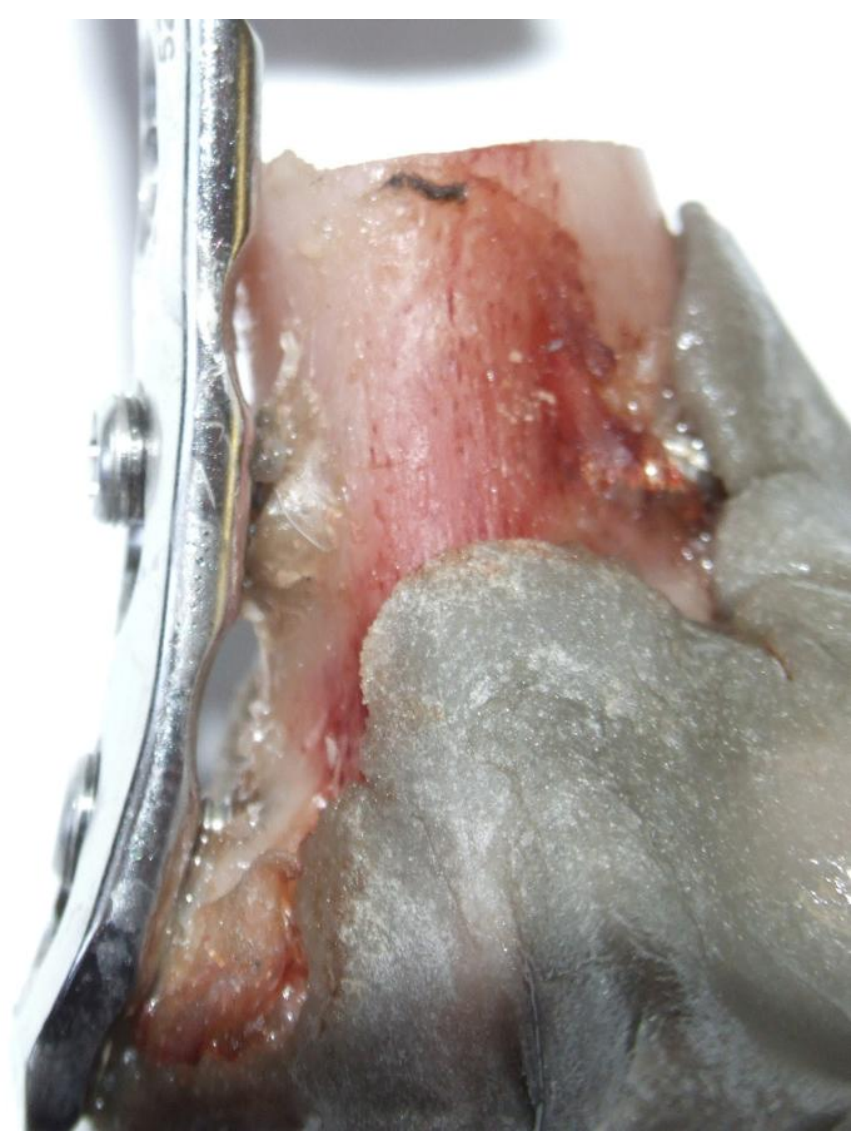

Figure 9: LCP construct showing incomplete seating of the proximal of the distal screw heads. 